\title{
Ornithine Carbamyl Transferase in Reye's Syndrome ${ }^{1}$
}

\author{
Devendra R. Deshmukh ${ }^{2}$ and Patrick L. Remington* \\ Department of Pediatrics and Communicable Diseases, University of Michigan, Ann \\ Arbor. Michigan 48109. and ${ }^{*}$ Michigan Department of Public Health, Lansing. \\ Michigan, and Field Services Division. CDC, Atlanta, Georgia
}

Received December 2, 1983

A recent report from our laboratory showed that in the serum of many Reye's syndrome (RS) patients, ornithine carbamyl transferase (OCT, Carbamyl phosphate: L-ornithine carbamyl transferase; EC 2.1.3.3.) activities were elevated during acute illness (1). In the present paper, serum OCT activities of acutely ill RS patients are compared with those of convalescent RS patients, their parents, and non-RS childhood control subjects, and the significance of serum OCT elevation in RS is discussed.

\section{METHODS}

The 47 serum samples of RS patients, taken during the acute illness were the residua of samples collected during the period 1974-1982 for diagnostic and related clinical examination. Thirty-seven of those samples were collected between 1974-1981, and serum OCT activities in those samples have been reported previously (1). The recovered RS patients were selected from cases reported to the Michigan Department of Public Health between January 1, 1980 and December 31, 1982. Only cases who were 5 years old or greater at the time of diagnosis and locatable residents of Michigan and who met the Centers for Disease Control (CDC) cases definition of stage II or greater RS were chosen (2). Serum specimens were also collected from the locatable biologic parents of the RS cases who were living in Michigan at the time of study. Of 42 eligible families, 41 agreed to partake in the study. The control specimens were taken from 47 school-age children from Jackson County, Michigan, who (with the approval of their parents) consented to provide blood samples for

'Supported in part by a grant from the National Institutes of Health, AI20236-0I.

2 To whom all correspondence should be addressed: F2815. Box 066, C.S. Mott Children's Hospital, University of Michigan Medical School, Ann Arbor, Mich. 48109. 
RS-related research, according to a protocol approved by the University of Michigan, Institutional Committee.

Blood specimens from the recovered RS patients and their parents were collected in acid citrate dextrose and centrifuged at $5000 \mathrm{~g}$ for 15 min. Plasma was separated and stored at $-20^{\circ} \mathrm{C}$. Serum OCT activity was determined by the method of Cerriotti (3) and expressed as units/ liter. Student's $t$ test was used to calculate the statistical significance. $P$ values $<0.05$ were considered significant.

\section{RESULTS}

Serum OCT values of 10 stage I and 37 stage II-IV RS patients are shown in Table 1. Serum OCT values for 7 of those stage I and 30 of stage II-IV RS patients were reported previously (1). The remaining three stage I patients and seven stage II-IV patients had serum OCT activities of $81.7 \pm 14.1$ and $18.1 \pm 3.1$, respectively (means \pm SEM). Serum OCT levels of acutely ill and convalescent RS patients are also shown in Table 1. The mean level in the convalescent RS patients and their parents were within normal range (0-10 units/liter) and were not significantly different from the controls.

\section{DISCUSSION}

Several reports indicate that the liver mitochondrial abnormalities, which are consistently seen in RS patients may be partially responsible for the metabolic disorders observed in this syndrome $(4,5)$. Decreased liver OCT activities have been reported in RS patients during the early phase of disorder (6). Our study suggests that the decrease in liver OCT activity was associated with an increase in serum OCT activity, because all the stage I patients had high serum OCT activities compared to stage II-IV patients and also compared to controls. The finding of normal

TABLE 1

Serum Ornithine Carbamyl Transferase Activities in Reye's Syndrome Patients and Their Parents

\begin{tabular}{|c|c|c|c|c|c|}
\hline \multirow[b]{2}{*}{ Controls } & \multicolumn{3}{|c|}{ Reye's syndrome patients } & \multicolumn{2}{|c|}{$\begin{array}{c}\text { Parents of } \\
\text { Reye's syndrome } \\
\text { patients }\end{array}$} \\
\hline & Stage I & Stage II-IV & Recovered & Fathers & Mothers \\
\hline $\begin{array}{c}6.3 \pm 0.5 \\
(47)\end{array}$ & $\begin{array}{c}91.4 \pm 15.5^{*} \\
(10)\end{array}$ & $23.5 \pm 2.3^{*}$ & $\begin{array}{c}7.7 \pm 0.3 \\
(44)\end{array}$ & $\begin{array}{c}9.5 \pm 0.5 \\
(25)\end{array}$ & $\begin{array}{c}8.5 \pm 0.3 \\
(29)\end{array}$ \\
\hline
\end{tabular}

Note. Results are expressed in units/liter as means $\pm \mathrm{SE}$ of the number of samples shown in parentheses. *Indicates statistically significant difference $(P<0.05)$ as compared with controls. 
serum OCT levels in the convalescent RS patients and in their parents (Table 1) suggest that an inherited, inborn metabolic defect was not responsible for the increased serum OCT activities in the RS patients. It is possible that the interactions of several exogenous factors, such as viral infection and drug consumption may have led to the increase in serum OCT activities in the RS patients during the early phase of the disorder. Studies in an animal model of RS have shown that the combination of influenza B viral infection, aspirin treatment, and diet-induced hyperammonemia produce significant increase in serum OCT activity (7).

Because RS is a rare disease, small number of cases were available for this study. In this report, serum OCT analysis of 10 additional RS patients supplemented and confirmed our earlier observation in $37 \mathrm{RS}$ patients that serum OCT activities are elevated in RS (1). This study also showed, for the first time that the convalescent RS patients and their parents have normal levels of serum OCT. Our earlier study showed that unlike SGOT activities, elevated serum OCT activities in RS patients correlate well with the serum ammonia levels and also with the neurologic stages of the disorder (1). Unlike SGOT, serum OCT is stable for a prolonged time and does not change due to hemolysis $(1,8)$. Because serum OCT elevation is more specific for liver mitochondrial damage, measurement of this enzyme may provide additional staging, diagnostic, and prognostic criteria for RS.

\section{SUMMARY}

Serum levels of ornithine carbamyl transferase activities were determined in the acutely ill and convalescent Reye's syndrome patients and in their parents. Acutely ill Reye's syndrome patients had elevated levels of serum ornithine carbamyl transferase activities as compared to those in controls. The convalescent Reye's syndrome patients and their parents had normal levels of serum ornithine carbamyl transferase activities. These results suggest that an inborn metabolic defect was not responsible for the increase in serum ornithine carbamyl transferase activities in Reye's syndrome.

\section{ACKNOWLEDGMENTS}

We thank William N. Hall, M.D. and Harry McGee, M.P.H., for providing the blood samples of the convalescent RS patients and their parents. We thank Dr. I. V. Baublis (deceased) for providing blood samples of the control subjects.

\section{REFERENCES}

1. Deshmukh, D. R., Rittenhouse, J. W., Mason, M.. and Baublis. J. V., Enzyme 27, 45 (1982).

2. Corey, L., Rubin, R., Bregman. D., and Gregg. M. B., Pediatrics 60, 702 (1977). 
3. Cerriotti, G., in "Clinical Biochemistry 2." (R. Curtius, Fd.1. p. 1151. de Gruyter Berlin, 1978.

4. DeVivo, D. C., Neurology 28, 105 (1978).

5. Thater, M. M., Amer. J. Dis. Child. 130, 241 (1976).

6. Brown. T., Hug, G.. Lansky, L.. Bove. K.. Scheve. A., Ryan, M., Brown. H., Schubert W. K., Partin. J. C. and Lloyd-Still. J., N. Engl. J. Med. 294, 861 (1976).

7. Deshmukh, D. R., Maassab, H. F., and Mason, M., Prox. Natl. Acad Sci. USA 79. 7557 (1982).

8. Reichard, H., and Reichard, P., J. Lab. Clin. Med. 52, 709 (1958). 